\title{
Infección por Cryptosporidium parvum en una mujer embarazada, inmunocompetente, con riesgo ocupacional
}

\author{
Patricia Neira O., Nelson Muñoz S. y M. José Rosales L.
}

\section{Cryptosporidium parvum infection in a pregnant inmunocompetent woman with occupational risk}

Cryptosporidioses is a parasitic zoonoses generated by diverse Cryptosporidium species. This coccidiosis affects multiple vertebrate species, including human beings. In Chile, as it happens in other countries, cryptosporidioses is a low frequency infection in immunocompetent individuals, acquiring a big relevance in immunocompromised ones. We present the following case: a recently graduated student from Veterinary medical school, with a 20 week pregnancy, living in "Laguna Verde" area in the Region of Valparaíso and who was infected with Cryptosporidium sp. Etiologic diagnosis was made by Ziehl Neelsen, and nested PCR followed by PCR product sequencing. During the same period, the infection was detected in her cats which were asymptomatic. In all of them, her and the cats, the species identified was Cryptosporidium parvum. Her husband and her other pets were all asymptomatic and non infected. This is the first report of a possible cryptosporidioses transmission between humans and cat.

Key words: Criptosporidiosis, Cryptosporidium parvum, nested PCR, zoonoses, occupational hazard profession, pregnancy.

Palabras clave: Criptosporidiosis, Cryptosporidium parvum, RPC anidada, zoonosis, riesgo profesional, embarazo.

\section{Introducción}

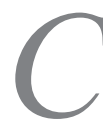

ryptosporidium parvum es un coccidio de importancia médica y veterinaria, que puede originar criptosporidiosis en el ser humano como en una gran variedad de especies vertebradas. La forma infectante de este coccidio, el ooquiste maduro, presenta una gran resistencia a los factores ambientales lo que le permite una amplia distribución en la naturaleza.

En el ser humano, como en otros animales, la infección se puede presentar en forma esporádica afectando a un solo individuo, o bien en forma de brote epidémico, situación que es infrecuente en Chile. Las especies más frecuentes en el ser humano, en el mundo y en Chile, son C. hominis (antroponótica) y C. parvum (zoonótica) ${ }^{1,2}$. Se han documentado algunas infecciones humanas por C. canis, C. felis, C. meleagridis, C. baileyi y C. muris ${ }^{3-9}$.

Los animales de compañía, en especial los perros y los gatos, constituyen probables fuentes de infección, ya que en ocasiones se infectan con C. parvum ${ }^{10}$. Actualmente, mediante estudios de biología molecular, se ha demostrado que la infección se produce mayoritariamente por $C$. canis y $C$. felis en perros y gatos respectivamente ${ }^{1,10}$. A pesar del estrecho contacto que se establece entre el ser humano y las mascotas (en especial perros y gatos), se considera excepcional la asociación de criptosporidiosis humana procedente de infecciones de las mascotas. Asimismo, ha sido difícil comprobar si son los animales los que infectan a su propietario. Existe sólo un reporte de la posible transmisión de $C$. canis, por cohabitación, entre dos hermanos y un perro en Lima-Perú ${ }^{11}$.

La patología humana puede darse en el contexto de un inmunocompromiso, principalmente en personas infectadas por VIH o enfermas de SIDA, u otras situaciones de inmunodeficiencia natural o adquirida. Entre los factores de riesgo se encuentra la profesión u ocupación ${ }^{12}$, pudiendo afectar a médicos veterinarios o estudiantes de esta carrera profesional, técnicos veterinarios, personal de laboratorio o personas que trabajan en contacto físico con los animales infectados como lo son los entrenadores y los trabajadores agrícolas. La exposición a aguas contaminadas, la ingestión de comida cruda o leche no pasteurizada y los viajes a zonas endémicas o rurales, constituyen potenciales situaciones de riesgo de infección.

A la fecha no ha sido publicado en Chile, otro caso clínico de criptosporidiosis en una mujer embarazada egresada de medicina veterinaria, diagnosticado etiológicamente mediante tinción de Ziehl Neelsen, e identificación de la especie mediante reacción de polimerasa en cadena (RPC) anidada (nested PCR) y la posterior secuenciación.
Universidad de

Valparaíso, Chile.

Facultad de Medicina Escuela de Medicina Depto. de Preclínicas Cátedra de Parasitología (PNO, NMS)

Universidad de Granada, España Facultad de Ciencias Instituto de Biotecnología Depto. de Parasitología (MJRL)

Financiado por Dirección de Investigación de la Universidad de Valparaíso (DIPUV 01/2005)

Recibido: 30 de julio de 2009 Aceptado: 8 de junio de 2010

Correspondencia a: Patricia Neira O. patricia.neira@uv.cl 
Figura 1. Ooquiste de Cryptosporidium spp de la paciente (a) y de cachorro de gato (b). Tinción de Ziehl Neelsen.
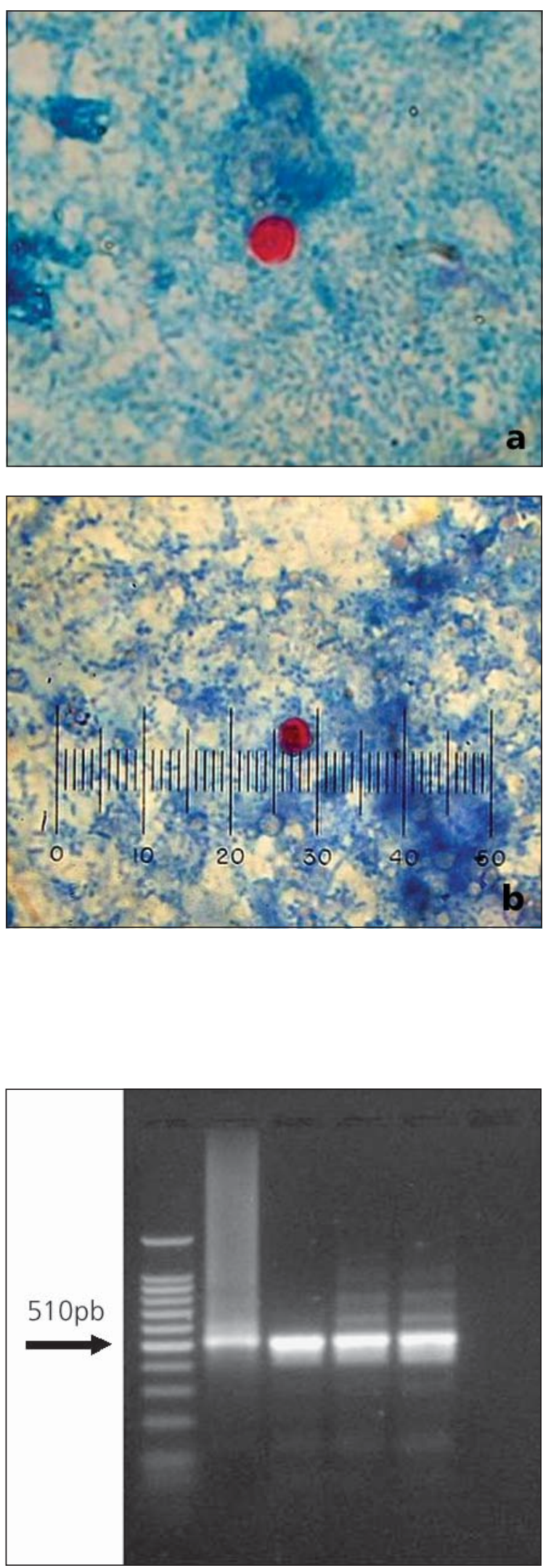

\section{Caso Clínico}

Paciente egresada de medicina veterinaria, con 28 años de edad, primípara, cursando un embarazo de 20 semanas, quien fuera derivada de un servicio de ginecología para el diagnóstico enteroparasitario de diarrea prolongada.

La paciente, procedente del sector de Laguna Verde (Región de Valparaíso), relataba una historia clínica de 14 días de evolución caracterizada por deposiciones líquidas, de 3 evacuaciones diarias, sin mucus ni sangre. Pensando en una fuente de infección, la enferma manifestó que en sitio eriazo vecino a su hogar, se había producido la ruptura de una cámara de alcantarillado. Su hogar poseía alcantarillado y agua de pozo. Refirió consumir verduras y frutas crudas, pero pescados y mariscos bien cocidos. Entre sus mascotas tenía cuatro perros (de 1 a 5 años), dos gatos de tres meses y una gata de un año; estos últimos se mantenían dentro del hogar y dormían en sofá y cama de la propietaria. La paciente trabajaba en una clínica de animales menores y su actividad consistía en vacunaciones, inyecciones y curaciones en esos animales.

Para el diagnóstico y estudio de la posible fuente de infección se solicitó muestras de heces a la paciente, su cónyuge y sus mascotas; además, muestra de agua del pozo de su casa y agua del sitio eriazo colindante a su hogar. Sólo la paciente presentaba diarrea, su cónyuge y sus mascotas se encontraban asintomáticos.

En el examen copro-parasitario mediante técnica de Telemann modificado no se observaron parásitos ni comensales. La tinción de Ziehl Neelsen de un extendido del centrifugado de la muestra fecal mostró la presencia de ooquistes de Cryptosporidium sp en la paciente y en los cachorros de gato (Figuras $1 \mathrm{a}$ y b) y en estos últimos la presencia de huevos de Dipylidium caninum. Las muestras fecales de su cónyuge, los perros, las muestras del agua de pozo y agua del sitio eriazo resultaron negativas para ooquistes de Cryptosporidium sp mediante la tinción de Ziehl Neelsen.

Para determinar la especie involucrada en la criptosporidiosis, las muestras fueron procesadas para diagnóstico mediante RPC anidada, de acuerdo a lo publicado ${ }^{2,13}$ y posteriormente sometidas a secuenciación bidireccional, identificándose en la paciente y sus gatos la presencia de C. parvum (Figura 2). Las secuencias fueron depositadas en GenBank, bajo los $\mathrm{N}^{\circ}$ de acceso: GQ355894 (gato), GQ355895 (mujer embarazada propietaria del gato). Las muestras de su cónyuge, perros $\mathrm{y}$ las muestras de agua del pozo y sitio eriazo resultaron negativas a la RPC.

Junto con el resultado, se indicó a la paciente medidas profilácticas y se la instruyó respecto al parásito y los mecanismos de transmisión. Se le solicitó exámenes de control. Como terapia, se le indicó hidratación y soporte sintomático. 
Los controles mediante método de Telemann modificado y tinción de Ziehl Neelsen a la paciente y a sus gatos, fueron efectuados a los 30 y 42 días de evolución. En el último control, la paciente acudió muy atemorizada pues en el hospital le informaron que el coccidio se transmite al feto vía transplacentaria y al buscar información en Internet sólo encontró que existe una asociación del parásito con el VIH/SIDA.

Debido a que la criptosporidiosis es una parasitosis que orienta a pensar en una infección por VIH y al hecho de que no habíamos detectado la presencia de Cryptosporidium sp en adultos, a excepción de pacientes enfermos de SIDA, a la paciente se le sugirió el examen mediante ELISA para VIH como medida preventiva, y según lo establecido por la Ley 19.996 para evitar la transmisión madre-hijo del virus, el que resultó negativo.

La gestante evolucionó en forma satisfactoria, sin diarrea y con clara mejoría del estado general a los 42 días del primer diagnóstico, con un examen coproparasitario, tinción de Ziehl Neelsen y RPC negativos. Su parto fue normal y sin complicaciones. Todas las mascotas fueron retiradas del interior de la vivienda.

\section{Discusión}

En Chile, la criptosporidiosis en los seres humanos es ocasionada principalmente por C. hominis, C. parvum ${ }^{2}$ $\mathrm{y}$, muy esporádicamente, por $C$. meleagridis $\left(\mathrm{N}^{\mathrm{o}}\right.$ acceso Genbank GQ865526) y C. muris ( $\mathrm{N}^{\mathrm{o}}$ acceso Genbank GU055303).

En individuos sanos, inmunocompetentes, C. parvum causa una diarrea auto-limitada, con una duración de 5 a 6 días $^{14}$. Entre los grupos de personas de mayor riesgo se encuentran los pacientes inmunocomprometidos como aquellos infectados con VIH o enfermos de SIDA, los que se encuentran en quimioterapia contra el cáncer, los sometidos a trasplantes de órganos sólidos o precursores hematopoyéticos y los que reciben corticoterapia. En riesgo potencial de infección por Cryptosporidium se encuentran las mujeres embarazadas ${ }^{15}$, a pesar de la normalidad de la mayoría de las funciones inmunitarias, lo que probablemente pueda deberse a la disminución en la inmunidad mediada por células para determinados antíge$\operatorname{nos}^{16,17}$. Sin embargo, se desconoce si durante el embarazo estas células son suficientes para controlar la intensidad de la criptosporidiosis. Existe muy poca información en la literatura en torno a los efectos de Cryptosporidium sobre el embarazo, tal vez debido a que muchos laboratorios, frente a la solicitud de un examen copro-parasitario en un paciente diarreico, no investigan rutinariamente las deposiciones para buscar la presencia de Cryptosporidium spp. Debido a la falta de antecedentes bibliográficos de criptosporidiosis en mujeres embarazadas, es difícil juzgar si la enfermedad es grave en este grupo de pacientes ${ }^{18}$. La infección se ha descrito en una mujer embarazada de 14 semanas y en su hijo, descartándose en su marido que trabajaba con aguas residuales ${ }^{18}$.

A partir de 1983 en gacela y posteriormente en 1995 en cordero, se demostró la infección intrauterina natural de los fetos por Cryptosporidium $\mathrm{spp}^{19,20}$. Estudios experimentales en ratones demostraron que es posible infectarlos iatrogénicamente con ooquistes inoculados en el aparato genital ${ }^{20}$. Se ha comprobado la parasitación intestinal fetal por el coccidio luego de infectar a hembras preñadas inmunodeprimidas de ratones ${ }^{21}$, lo que llevaría a pensar en el paso transplacentario del parásito al feto.

No existiendo evidencia científica al respecto, si el mecanismo llegase a producirse en humanos implicaría un riesgo de infección fetal transplacentaria. Pero también la infección del neonato se puede explicar en su paso a través del canal del parto por la cercanía de los genitales externos con el orificio anal.

Si bien la transmisión por fecalismo directo constituye un riesgo para cualquier ser humano, debido al trabajo que desempeñan los médicos veterinarios, los cuidadores y/o manipuladores de animales, los entrenadores, los agricultores y los propietarios de mascotas, por su actividad y contacto, se encuentran más expuestos a la infección.

El rol que cumplen los animales de compañía en la transmisión del coccidio a los seres humanos aún permanece sin dilucidar. La asociación entre la ocurrencia de la criptosporidiosis pediátrica y el contacto con perros y gatos se ha comunicado en Guinea-Bissau e Indonesia ${ }^{22,23}$. En Inglaterra, el contacto con perros y gatos no se considera como un factor de riesgo de la criptosporidiosis ${ }^{24}$, y en Australia el contacto con mascotas se considera un factor de protección de la salud ${ }^{25}$.

Los recientes estudios de epidemiología molecular han demostrado que los gatos se infectan casi exclusivamente con $C$. felis ${ }^{10}$, y que la mayoría de las infecciones en los seres humanos se producen por C. parvum y C. hominis . $^{1,2}$. El rol de los gatos en la transmisión de la criptosporidiosis podría ser limitado. Sin embargo, en este caso clínico la identificación en el mismo periodo de la misma especie (C. parvum) (Figura 2) en la ama como en las mascotas, permite plantear con evidencia que este sería el primer caso en demostrar la transmisión zoonótica en nuestro medio.

Apoya lo anterior la ausencia del parásito en su marido y las otras mascotas, así como en el agua contaminada del peridomicilio. Sin embargo, su trabajo con animales menores pudo ponerla en riesgo de infectarse con el coccidio a partir de ellos, pero la probabilidad de que ella haya infectado a sus gatos es mucho menor que la situación contraria: los gatos (vagabundos y recogidos) se habrían infectado en el ambiente externo transmitiéndolo a la paciente, dada las precarias precauciones para evitar 
las infecciones de transmisión fecal (como el dormir en el sofá y cama de la paciente).

En los gatos pueden desarrollarse infecciones subclínicas y es posible que transmitan el coccidio a los seres humanos, en especial cuando son cachorros ${ }^{10}$, como en este caso.

Las enfermedades zoonóticas son uno de los riesgos que enfrenta el personal veterinario en sus actividades diarias $^{26}$, siendo más susceptible a la infección por agentes zoonóticos y con la posibilidad de desarrollar complicaciones más graves, el personal inmunocomprometido. La respuesta inmune puede estar suprimida, entre otras, por algunas de las siguientes condiciones: infección por el $\mathrm{VIH}$, diabetes mellitus, ciertas enfermedades malignas o inmunodeficiencias congénitas, la desnutrición, los tratamientos quimioterápicos, los fármacos inmunosupresores, los corticosteroides, la radiación y el embarazo.

Entre las infecciones zoonóticas parasitarias adquiridas ocupacionalmente se incluye la criptosporidiosis en estudiantes de medicina veterinaria ${ }^{12,27,28}$. En ellos la transmisión del coccidio por fecalismo directo puede ocurrir cuando manipulan a los animales durante el examen, el baño, la administración de tratamientos curativos o profilácticos, las caricias o bien a través del contacto con fomites (como por ejemplo las jaulas) contaminados con heces $^{29}$, sin tener la posterior precaución de un adecuado lavado de manos. Además de la inmunidad adquirida y específica, los adultos de varias especies aparentemente desarrollan una resistencia innata a la criptosporidiosis dependiente de la edad, por lo cual, los animales jóvenes son probablemente más importantes como fuente de infección que los animales adultos. Se han descrito casos en estudiantes de medicina veterinaria en contacto directo con heces de terneros infectados en forma natural o experimental ${ }^{12}$. La diarrea en los terneros facilita la transmisión zoonótica de criptosporidiosis, por lo que es necesario que las personas expuestas adopten estrictas medidas higiénicas que incluyen el uso de guantes y ropa de protección cuando se trabaja en un ambiente potencialmente contaminado con Cryptosporidium sp.

Los fomites constituyen un medio para la transmisión del coccidio. En un brote se confirmó un caso humano que tuvo contacto con ropa sucia con material fecal de terneros infectados ${ }^{30}$. Los ooquistes de Cryptosporidium son resistentes a la desecación y a varios desinfectantes y por lo tanto puede persistir en el ambiente.

El diagnóstico del coccidio, se efectúa con el hallazgo de los ooquistes en heces que deben ser estudiadas con la tinción de Ziehl Neelsen por su característica de ácido alcohol resistencia que los destaca del detritus fecal. El informe deberá indicar la presencia de ooquistes de Cryptosporidium spp, debido a que es imposible diferenciar la forma infectante (ooquistes) de las diferentes especies, sólo por las características morfométricas.
La determinación de la especie involucrada en la infección (20 validadas a la fecha, de las cuales nueve afectan a los seres humanos), sólo se logra a través de técnicas moleculares, siendo buenas herramientas la RPC anidada, acoplada al polimorfismo de la longitud de los fragmentos de restricción (RFLP, por su sigla en inglés). Orientan la sospecha diagnóstica los antecedentes epidemiológicos (contacto con fomites y ambientes potencialmente contaminados con heces humanas o de animales infectados).

No existe un tratamiento específico para la criptosporidiosis. Nitazoxanida ha demostrado ser eficaz sólo en algunos casos y está aprobada por la FDA para su uso en el control de esta coccidiosis. Este medicamento no se encuentra disponible en nuestro país y no se recomienda su uso durante el embarazo, por lo tanto, el tratamiento estará basado en medidas de soporte como rehidratación oral o intravenosa si la pérdida de líquido es importante, el reemplazo de electrólitos y el uso de agentes antiespasmódicos.

La naturaleza autolimitada de la enfermedad en individuos inmunocompetentes generalmente es una razón suficiente para no usar terapia específica, excepto en circunstancias excepcionales. Es también una buena práctica no administrar quimioterapia a mujeres embarazadas a menos que sea absolutamente necesario, como ocurriría si se llegara a comprobar la transmisión congénita de este parásito durante la gestación.

Frente a los hallazgos experimentales y relatados, y a no poder descartar que ello no se produzca también en humanos, cabría tomar medidas que eviten la transmisión fecal de infecciones durante el embarazo, especialmente en madres inmunodeprimidas, entre las cuales se debe mencionar el lavado de manos luego de haber mantenido contacto con animales, antes de llevárselas a la boca, antes de comer y previo a la manipulación de alimentos.

El ser humano propietario de las mascotas debería administrar alimentos libres de infección y ocupar en ellos utensilios que no sean de uso humano; evitar el contacto de la mascota con deposiciones humanas y animales y la adecuada eliminación de ellas, procurando utilizar guantes. Evitar el contacto de la cara con el animal, y el hábito de besarlos. Evitar que el animal descanse en sillas, sillón, cama. El baño de la mascota no debe efectuarlo una mujer embarazada.

En resumen, este caso corresponde al primero en Chile, en relación a la posible transmisión de C. parvum entre vertebrados (humano y mascota) que cohabitan; relacionado con embarazo y actividad profesional de mayor riesgo de infección (egresada de medicina veterinaria).

Agradecimientos: A Mario Párraga, al Sr. Ivo Carrasco por su apoyo en las técnicas moleculares y secuenciación de las mismas. 


\section{Resumen}

La criptosporidiosis es una zoonosis parasitaria provocada por diversas especies de Cryptosporidium. Esta coccidiosis afecta a múltiples vertebrados, incluido el ser humano. En Chile, al igual que en otros países, es una infección poco frecuente en inmunocompetentes y adquiere gran relevancia en pacientes inmunocomprometidos. Se presenta el caso de una egresada de la carrera de Medicina Veterinaria, embarazada, con 20 semanas de gestación, procedente del sector de Laguna Verde, Región de Valparaíso, que fuera infectada por Cryptosporidium sp. El diagnostico etiológico se realizó con tinción de Ziehl Neelsen, RPC anidada y posterior secuenciación. En el mismo periodo se detectó la infección en sus gatos asintomáticos. En ella y los animales se identificó a $C$. parvum. Su cónyuge así como sus otras mascotas no estaban infectados. Este corresponde al primer reporte de una posible transmisión de criptosporidiosis entre ser humano y gato.

\section{Referencias}

1.- Xiao L, Ryan U M. Cryptosporidiosis: an update in molecular epidemiology. Curr Opin Infect Dis 2004; 17:483-90.

2.- Neira-Otero P, Muñoz-Saldías N, SánchezMoreno M, Rosales-Lombardo M J. Molecular characterization of Cryptosporidium sp. and genotypes in Chile. Parasitol Res 2005; 97: 63-7.

3.- Xiao L, Bern C, Limor J, Sulaiman I, Roberts J, Checkley W, et al. Identification of 5 types of Cryptosporidium parasites in children in Lima, Perú. J Infect Dis 2001; 183: $492-7$.

4.- Akiyoshi D, Dilo J, Pearson C, Chapman S, Tumwine J, Tzipori S. Characterization of Cryptosporidium meleagridis of human origin passaged through different host species Infect Immun 2003; 71 (4): 1828-32.

5.- Coupe S, Sarfati C, Hamane S, Derouin F. Detection of Cryptosporidium and identification to the species level by nested PCR and restriction fragment length polymorphism. J Clin Microbiol 2005; 43: 1017-23.

6.- Leoni F, Gallimore CI, Green J, McLauchlin J. Characterisation of small double stranded RNA molecule in Cryptosporidium hominis, Cryptosporidium felis and Cryptosporidium meleagridis. Parasitol Int 2006; 55 (4): 299-306.

7.- Ditrich O, Palkovi L, Sterba J, Prokopic J, Loudova J, Giboda M. The first finding of Cryptosporidium baileyi in man. Parasitol Res 1991; 77: 44-7.

8.- Palmer C, Xiao L, Terashima A, Guerra H, Gotuzzo E, Saldías G, et al. Cryptosporidium muris, a rodent pathogen, recovered from a human in Perú. Emerg Infect Dis 2003; 9 (9): 1174-76.

9.- Cacciò S, Pinter E, Fantini R, Mezzaroma I, Pozio E. Human infection with Cryptosporidium felis: Case report and literature review. Emerg Infect Dis 2002; 8: 85-6.

10.- Santín M, Trout J. Companion animals. En: Fayer R, Xiao L. Cryptosporidium and cryptosporidiosis. Boca Raton, FL. CRC Press 2008; pp.437-50.

11.- Xiao L, Cama V, Cabrera L, Ortega Y, Pearson J, Gilman R. Possible transmission of Cryptosporidium canis among children and a dog in a household. J Clin Microbiol 2007; 45 (6): 2014-6.

12.- Levine J F, Levy M G, Walker R L, Crittenden S. Cryptosporidiosis in veterinary students. J Am Vet Med Assoc 1988; 193 : 1413-4.

13.- Neira P. Acerca de Cryptosporidium spp en Chile. Rev Méd Chile 2005; 133: 847-9.

14.- Nichols G. Epidemiology. En: Fayer R, Xiao L. Cryptosporidium and Cryptosporidiosis. Boca Raton, FL. CRC Press 2008; pp 79-118.

15.- Hoveyda F, Davies W A R, Hunter P R. A case of cryptosporidiosis in pregnancy. Eur J Clin Microbiol Infect Dis 2002; 21: 637-8.

16.- Gehrz R C, Christianson W R, Linner K M, Conroy M M, Mc Cue S A, Balfour H H Jr. A longitudinal analysis of lymphocyte proliferative responses to mitogens and antigens during human pregnancy. Am J Obstet Gynecol 1981; 140: 665-70.

17.- Weinberg E D. Pregnancy associated depression of cell-mediated immunity. Rev Infect Dis1984; 6: 814-31.

18.- Leek S. Case study: cryptosporidiosis complicating pregnancy and the puerperium. Midwives Chron 1987; 100: 72-4.

19.- Fleta J, Sánchez-Acedo C, Clavel A, Quílez J. Detection of Cryptosporidium oocysts in extra-intestinal tissues of sheep and pigs. Vet Parasitol 1995; 9 (3-4): 1-205.

20.- Liebler E M, Pohlenz J F, Woodmansee D B. Experimental intrauterine infection of adult BALB/c mice with Cryptosporidium sp. Infect Immun 1986; 54 (1): 255-9.

21.- Kanyari P W N, Oyejide A O, Alak J I B, Anderson D L, Wilson ST, Srivastava K. Cryptosporidium parvum: experimental transplacental transmission in murine hosts. Israel J Vet Med 2002; 57 (2): http://www. isrvma.org/article/57 2 3.htm)

22.- Molbak K, Aaby P, Hojlyng N, da Silva A P. Risk factors for Cryptosporidium diarrhea in early childhood: a case-control study from Guinea-Bissau, West Africa. Am J Epidemiol 1994; 139: 734-40.

23.- Katsumata T, Hosea D, Wasito E, Kohno S, Hara K, Soeparto P, et al Cryptosporidiosis in Indonesia: a hospital-based study and a community-based survey. Am J Trop Med Hyg 1998; 59: 628-32.

24.- Goh S, Reacher M, Casemore D P, Verlander N Q, Chalmers R, Knowles M, et al. Sporadic cryptosporidiosis, North Cumbria, England, 1996-2000. Emerg Infect Dis 2004; 10: $1007-15$.

25.- Robertson B, Sinclair M I, Forbes A B, Veitch M, Kirk M, Cunliffe D, et al. Case-control studies of sporadic cryptosporidiosis in Melbourne and Adelaide, Australia. Epidemiol Infect 2002; 128: 419-31.

26.- Langley R L, Pryor W H, O'Brien K F. Health hazards among veterinarians: a survey and review of the literature. J Agromedicine 1995; 2: 23-52.

27.- Preiser G, Preiser L, Madeo L. An outbreak of cryptosporidiosis among veterinary science students who work with calves. J Am Coll Health 2003; 51: 213-5.

28.- Gait R, Soutar R H, Hanson M, Fraser C, Chalmers R. Outbreak of cryptosporidiosis among veterinary students. Vet Rec 2008; 162 (26): 843-5.

29.- Siegel J D, Rhinehart E, Jackson M, Chiarello L. 2007 Guideline for isolation precautions: preventing transmission of infectious agents in healthcare settings, June 2007. Disponible en: www.cdc.gov/ncidod/dhqp/pdf/guidelines/ Isolation2007 (accedido 22 de abril 2009).

30.- Reif J S, Wimmer L, Smith J A, Dargatz D A, Cheney JM. Human cryptosporidiosis associated with an epizootic in calves. Am J Public Health 1989; 79: 1528-30. 